\title{
Astrometric precision of observations at VLT/FORS2
}

\author{
P. F. Lazorenko
}

\author{
Main Astronomical Observatory, National Academy of Sciencies of the Ukraine, Zabolotnogo 27, 03680 Kyiv-127, Ukraine \\ e-mail: laz@mao.kiev.ua
}

Received 23 September 2005 / Accepted 24 November 2005

\section{ABSTRACT}

In this paper we test the astrometric precision of VLT/FORS2 observations using a series of CCD frames taken in the Galactic bulge area. A special reduction method based on symmetrization of reference fields was used to reduce the atmospheric image motion. The positional precision of unsaturated $R=16 \mathrm{mag}$ star images at a $17 \mathrm{~s}$ exposure and $0.55^{\prime \prime}$ seeing was found to be equal to $300 \mu$ as. The total error of observations was decomposed into components. It was shown that astrometric error depends mainly on the photon centroiding error of the target ( $250 \mu$ as for 16 mag stars), while the image motion was much less (110 $\mu$ as). At galactic latitudes to about $20^{\circ}$, precision for a series of frames with a 10 min total exposure was estimated to be 30-50 $\mu$ as for 14-16 mag stars, providing the images were not overexposed and the filter $R_{\text {special }}$ was used. Error estimates for fields with smaller sky star density are given. We conclude that astrometric observations with large telescopes, under optimal reduction, are never atmospheric limited. The bias caused by differential chromatic refraction and the residual chromatism of LADC are considered and expressions that are valid for correcting color effects in the measured positions are given.

Key words. astrometry - methods: data analysis - atmospheric effects

\section{Introduction}

The studies of exoplanets and microlensing effects are usually restricted to measuring two physical quantities: radial velocities and/or brightness of stars. Angular measurements are rarely considered due to their poor precision. The extraction of many parameters related to the studied object from a single physical quantity, however, is not always possible or even reliable, so that the radial velocity data only allows us to find the low limit of planet mass. The additional information on the star angular displacements essentially improves the accuracy of determining exoplanet mass and orbital parameters (Pravdo \& Shaklan 1996; Benedict et al. 2002). These data are also useful for microlensing studies of massive compact objects in the galaxy because they enables us to measure lens mass, its distance, and velocity (e.g. Boden et al. 1998). Safizadeh et al. (1999) argue that a combined use of photometric microlensing and astrometric measurements allows the detection of low mass planets and determination of their masses and semi-major axes.

To be useful, the astrometric measurements need to be an accurate for these purposes, at least on the order of 10 to $100 \mu$ as. The long history of ground-based observations shows, however, that the actual accuracy is in fact much lower, about 1 mas per a night for a 10-20' reference frame (Gatewood 1987) and $1 \mathrm{mas} / \mathrm{h}$ for a $1^{\prime}$ double-star separation measurement (Han 1989). The best $150 \mu \mathrm{as} / \mathrm{h}$ precision was achieved by Pravdo \& Shaklan (1996) in a special series of observations at the 5-m Palomar telescope. A major factor limiting accuracy is the image motion caused by atmospheric turbulence and displayed as a random relative change of star-image positions in unpredicted directions and at an uncertain angle. Therefore development of ultra high-precision astrometric methods of observations from the ground is normally related to infrared interferometers that have $10 \mu$ as expected precision (e.g. Frink et al. 2004). A similar accuracy, at acceptable exposure times, was found to be unattainable for filled one-aperture telescopes due to atmospheric image motion (Lindegren 1980).

This conclusion was revised in our previous work (Lazorenko \& Lazorenko 2004, hereafter Paper I) where we considered the image motion as a turbulent light phase-related quantity dependent on initial wave-front fluctuations at the telescope entrance pupil, on the process of differential measurements, and on the way these phase fluctuations affect the measured positions. In the spectral domain, transformation of the phase fluctuations into image motion is described by four filters of non-atmospheric origin that correspond to: 1) conversion from the phase to the wave-front gradient; 2) averaging over the entrance pupil; 3) averaging due to a finite exposure; and 4) formation of function differences in the directions to reference stars. Favoring the inhibition of the atmospheric image motion spectrum is the circumstance that a filter $Y(q)$ corresponding to the averaging over the entrance pupil is a low-pass filter that is transparent to about $q \sim 2 / D$ spatial frequency ( $D$ is the telescope diameter), while reference field stars form a high-pass filter $Q(q)$. The filter bands are only partially overlapping by their rising (for $Q(q)$ ) and descending (for $Y(q)$ ) branches. The shape 
of these instrumental functions can be adjusted so as to minimize the combined system response $Y(q) Q(q)$. Improvement of the $Y(q)$ filter shape is achieved by apodization or by applying a special covering with a variable light transmission to the entrance pupil. This measure results in a fast attenuation of the filter transmission at $q>2 / D$. The function $Q(q)$ shape is adjusted at the reduction phase by setting special weights to each reference star, which virtually reconfigures the reference field into a highly symmetric star group and suppresses $Q(q)$ response at low frequencies.

The attenuation factor of the system with modified filters $Y(q)$ and $Q(q)$ depends on the telescope diameter, angular reference field radius $R$, and the turbulent layer height $h$, and being a power function of the $R h / D$ ratio, is especially large for narrow-field mode of observations when $R h \ll D$. For that reason, the method is recommended for future large 30-100 m telescopes, although the expected precision is also high for existing $8-10 \mathrm{~m}$ instruments. Based on theoretical assumptions, we showed that for $10-\mathrm{m}$ telescopes precision of differential referencing to the background stars is, depending on the brightness and number of reference stars, from 10 to $60 \mu$ as at $10 \mathrm{~min}$ exposure.

In the current study we test the effeciency of this astrometric method application to actual observations at the VLT and in the presence of numerous noise sources, such as atmospheric image motion, photon noise in the images of stars, pixelization, complex shape of PSF, background noise, optical aberrations, active optics performance, etc. In Sect. 6 we discuss another atmospheric effect known as differential chromatic refraction (DCR), which causes a relative displacement of star images of different colors (Pravdo \& Shaklan 1996; Monet et al. 1992; Louarn et al. 2000). At the VLT, the DCR effect is strongly reduced by use of a special longitudial atmospheric dispersion compensator (LADC) that mimics atmospheric refraction but introduces it in the opposite direction (Avila et al. 1997).

\section{Observational data and computation of centroids}

For the test study we used a 4-hour series of FORS2 frames obtained by Moutou et al. (2004) when observing OGLE-TR$132 \mathrm{~b}$ object with the filter $R_{\text {special }}$ at $17 \mathrm{~s}$ average exposure and a good $F W H M=0.55^{\prime \prime}$ seeing. The HR mode with $2 \times 2$ pixel binning and $0.125^{\prime \prime}$ pixel size was used. Only the master chip of CCD with a field of view of $3.9 \times 2.1^{\prime}$ containing about 400 stars brighter than $20 \mathrm{mag}$ was considered, and only well-exposed stars fainter than $16 \operatorname{mag}(R)$ were measured. Four frames obtained with a $V$ filter provided color information that was then used for examination of chromatic effects (Sect. 6).

A full profile fitting was used for computation of $\bar{x}, \bar{y}$ coordinates of stellar centroids. The shape of the PSF was fitted by a 12 parameter model of a sum of three elliptic Gaussians with a common $\bar{x}, \bar{y}$ center:

$$
\begin{aligned}
\operatorname{PSF}(x, y)= & I G(x, y)+(x-\bar{x})^{2} I^{\prime} G^{\prime}(x, y) \\
& +(y-\bar{y})^{2} I^{\prime \prime} G^{\prime \prime}(x, y)
\end{aligned}
$$

where $x, y$ are pixel coordinates. The main Gaussian $G$ with 5 free parameters $\left(\bar{x}, \bar{y}\right.$, Gaussian width parameters $\sigma_{0 x}, \sigma_{0 y}$, a term $\alpha_{0}$ specifying orientation of semi-axes) and a flux $I$ (sixth parameter) contains about $98-99 \%$ of the total star flux; two auxiliary Gaussians $G^{\prime}$ and $G^{\prime \prime}$ with fluxes $I^{\prime}$ and $I^{\prime \prime}$ are oriented along $x, y$ axes. As model parameters, they both include $\bar{x}, \bar{y}$, two width terms $\sigma_{1 x}, \sigma_{1 y}$ for $G^{\prime}$ and similar $\sigma_{2 x}, \sigma_{2 y}$ terms for $G^{\prime \prime}$. Note that since image motion affects photocenter positions, it is useful to treat $\bar{x}, \bar{y}$ in Eq. (1) not as the "fitting model center" but as the weighted photocenter defined by equations $\iint(x-\bar{x}) P S F(x, y) \mathrm{d} x \mathrm{~d} y=\iint(y-\bar{y}) P S F(x, y) \mathrm{d} x \mathrm{~d} y=0$. This interpretation emphasizes the necessity of using the PSF models, with first derivates on model parameters (except $\bar{x}, \bar{y}$ ) being symmetric functions of coordinates $x, y$. In this case small errors in the parameter determination induce a symmetric bias of the PSF shape that does not affect computation of $\bar{x}, \bar{y}$. For that reason, the model (1) does not contain odd coordinate powers.

Transition from the continuous expression for the number of electrons (1) to the discrete pixel counts requires numerical integration of $\operatorname{PSF}(x, y)$ within the pixel limits. To avoid this procedure, we used analytical approximation of the integral precise to $10^{-4}$.

The solution of Eq. (1) was obtained in square $10 \times$ $10 \mathrm{px}$ windows that contained the main light signal and are small enough to minimize overlapping of nearby images. Approximation of the actual PSF shape by the model (1) is typically accurate to $5-10 \%$, while the photon noise statistics differs essentially from the Poissonian. Deviations in the actual PSF shape from the model have a specific wavelike pattern that is highly correlated for all stars' images of a certain frame (especially at short spatial scales) but completely changes at the next frame. At a high level of a light signal (bright images, central pixels), these deviations strongly exceed random photon noise.

Using an initial fitting for each star, we averaged the "measured PSF-model" residuals over all star images in a frame to find the systematic part of these residuals as a function $\operatorname{var} P S F(x-\bar{x}, y-\bar{y})$. To obtain this function estimates at subpixel level, it was approximated by a set of $2 \mathrm{D}$ cubic polinomials defined in $3 \times 3$ pixel areas with centers displaced at 1 pixel. The function $\operatorname{var} P S F(x-\bar{x}, y-\bar{y})$ was then subtracted from individual star images and a final profile fitting applied. This slightly improved the precision of the centroiding. One can see that the measured PSFs usually show variations at spatial scales of some hundreds of pixels. Therefore it seems reasonable to obtain more precise $\operatorname{var} P S F(x-\bar{x}, y-\bar{y})$ function shapes by performing averaging of the "measured PSF-model" residuals only in the limited areas of a frame. Although leading to much better fitting of PSFs, this consideration is incorrect with regard to determining the photocenters because application of variable image shape corrections dependent on position of a star in the frame induces a space-variable bias in the weighted center positions.

An attempt to assign weights to pixels depending on these residual values also failed, although the quality of PSF approximation according to $\chi^{2}$ criterion improved. This criterion seems to be improper for precise centroiding works since the actual centroiding precision is near the photon limit (Sect. 4) despite of low $\chi^{2} \sim 10$ fitting quality for bright stars. 
To estimate the centroiding precision for images with PSFs of a complex shape (1), we created random images adding Poisson noise to the model function. At the $340 \mathrm{e}^{-} / \mathrm{px}$ sky level typical of FORS2, the results are represented by the expression

$\varepsilon=\frac{F W H M}{2.26 \sqrt{I}}\left(1+475 I^{-0.7}\right)$.

Here $F W H M$ refers to the $x$ or $y$ axis and $I$ is given in electrons. Note that Eq. (2) is valid for random photon fluctuations only and does not take the real photon statistics in central pixels into account. It was found (Sect. 4.1) that for bright images a limit (2) is not achievable.

\section{Reduction model}

Astrometric reductions are based on the theoretical considerations in Paper I whose main results are reproduced here in a concise form.

The variance $\sigma_{\text {at }}^{2}$ of differential atmospheric image displacements caused by a thin turbulent layer can be found by integration of the power spectrum of image motion which, as mentioned in the Introduction, contains filter functions $Y(q)$ and $Q(q)$. Therefore, using Eq. (12) from Paper I, we obtain

$\sigma_{\text {at }}^{2}=\frac{J(h)}{V T} \int_{0}^{\infty} Y(q)\left[\sum_{m=1}^{\infty} q^{2 m} F_{2 m}\left(x_{i}, y_{i}\right)\right] q^{-5 / 3} \mathrm{~d} q$

where $T$ is the exposure, $V$ the velocity of the turbulent layer, and $J(h)$ a function proportional to the intensity of turbulence in the layer and its thickness. The integration is performed in the area of a $2 \mathrm{D}$ spatial frequency $q$ related to the turbulent layer plane. Cartesian star coordinates $x_{i}, y_{i}$, not distorted by the atmosphere, refer to the same plane. The subintegral component in square brackets represents the $Q(q)$ function and describes the filtration caused by the reference star field. In its original form (Eq. (25) of Paper I), it is written as a linear combination of Bessel zero-order functions dependent on the star-coordinate differences. Note that the $Q(q)$ component is expanded into $q^{2 m}$ powers with coefficients $F_{2 m}$, which are functions of $2 m$ order cross-moments of star coordinates $x_{i}, y_{i}$ differences measured with reference to the target. The amplitude of the $q^{2 m} F_{2 m}$ components rapidly decreases with $m$. The $Y(q)$ function describes the filtration of the original image motion spectrum by the telescope entrance pupil, which can be apodized for better inhibition of high-frequency spectral components; for a filled pupil, an expression for $Y(q)$ is given by Eq. (13) of Paper I.

It is easy to formulate the conditions at which the principal component $F_{2}$ and possibly some subsequent $F_{2 m}$ functions turn to zero at the reduction stage. For that purpose, it is necessary to define the target position with reference to $N$ field stars by the quantity (Eq. (19) of Paper I)

$W=N^{-1} \sum_{i=1} a_{i}\left(\bar{x}_{0}-\bar{x}_{i}\right)=\bar{x}_{0}-N^{-1} \sum_{i=1} a_{i} \bar{x}_{i}$

for the $x$-axis and by a similar quantity for the $y$-axis. Here $\bar{x}_{i}$, $\bar{y}_{i}$ are the measured (image motion included) Cartesian coordinates of reference stars $i=1,2 \ldots N$; index $i=0$ refers to the target. Weights $-\infty<a_{i}<\infty$ are determined from conditions

$$
\begin{aligned}
& \sum a_{i}\left(x_{i}-x_{0}\right)^{\alpha}\left(y_{i}-y_{0}\right)^{\beta}=0, \alpha+\beta=1 \ldots \frac{k}{2}-1, \\
& \sum a_{i}=N, \sum a_{i}^{2} \varepsilon_{i}^{2}=\min
\end{aligned}
$$

where $\alpha$ and $\beta$ are non-negative integers, and $\varepsilon_{i}$ is the centroiding accuracy for $i$ th star. The first equation in (5) formally describes a system of points with zero-weighted cross-moments of the $k / 2-1$ order and the symmetry center at $x_{0}, y_{0}$. The even parameter $k=2,4 \ldots$ is therefore called the reference field symmetry order and is related to the power of the first $q^{k} F^{k}$ non-zero component of Eq. (3). The last condition in (5) is useful for minimizing the reference field centroid errors that affect the accuracy of $W$ positions. The system (5) is solved when at least $N_{\min }=k(k+2) / 8$ reference stars are available.

In the simplest case of $k=2$, the first condition of the system (5) is not used and weights are found from equations $\sum a_{i}=N, \sum a_{i}^{2} \varepsilon_{i}^{2}=\min$. This refers, for example, to the double star $(N=1)$ separation $W_{x}=\left(\bar{x}_{0}-\bar{x}_{1}\right)$ measurements or to the average of individual separations $W_{x}=N^{-1} \sum\left(\bar{x}_{0}-\bar{x}_{1}\right)$, if $N>1$ reference stars of equal brightness are available. The field is not symmetrized, and the $\sigma_{\text {at }}^{2}$ value is maximum since the largest $q^{2} F_{2}$ term in Eq. (3) is not eliminated.

The first $k=4$ symmetrized solution is obtained by applying conditions $\sum a_{i}\left(x_{i}-x_{0}\right)=\sum\left(y_{i}-y_{0}\right)=0$. In particular, these conditions refer to the continuous distribution of reference stars in a circular field that, as shown by Lindegren (1980), yields a strong inhibition of the image motion. The same $k=4$ symmetry order with elimination of the $q^{2} F_{2}$ term can be implemented with use of only 3 arbitrary placed stars. The principal term in Eq. (3) is proportional to $q^{4} F_{4}$.

The highest $k=12$ order implementation used in this study requires at least $N_{\min }=21$ reference stars to apply conditions $\sum a_{i}\left(x_{i}-x_{0}\right)=\sum a_{i}\left(y_{i}-y_{0}\right)=\sum a_{i}\left(x_{i}-x_{0}\right)^{2}=\sum a_{i}\left(x_{i}-x_{0}\right)\left(y_{i}-\right.$ $\left.y_{0}\right)=\ldots=\sum a_{i}\left(x_{i}-x_{0}\right)\left(y_{i}-y_{0}\right)^{4}=\sum a_{i}\left(y_{i}-y_{0}\right)^{5}=0$. This results in elimination of $F_{2}, F_{4}, F_{6}, F_{8}$, and $F_{10}$ terms, thus $Q(q)$ is proportional to $q^{12} F_{12}$.

In proper motion works, the displacement $\mu_{x}$ of the target star is found as a difference of $W(\mathrm{Ep} 2)-W(\mathrm{Ep} 1)$ at epochs Ep2 and Ep1. Weights $a_{i}$ are determined from conditions (5) in which the measured positions at Ep1 (standard frame) are used as unknown true coordinates $x_{i}=\bar{x}_{i}(\mathrm{Ep} 1), y_{i}=\bar{y}_{i}(\mathrm{Ep} 1)$. This substitution, as seen from Eqs. (5), leads to the identity $W($ Ep1 $)=0$ that defines a conditional zero-point of $W$ values. Positions $W($ Ep1) also define a zero-point of proper motions which are computed as

$$
\begin{aligned}
\mu_{x} & =W(\operatorname{Ep} 2)=\frac{1}{N} \sum a_{i}\left(\bar{x}_{0}-\bar{x}_{i}\right) \\
& =\bar{x}_{0}-x_{0}+\frac{1}{N} \sum a_{i}\left(x_{i}-\bar{x}_{i}\right)
\end{aligned}
$$

Symmetrization of reference fields not only suppresses atmospheric image motion, but also reduces geometrical field distortions, in this respect being equivalent to the usual reduction models. Let us consider astrometric reduction model based on 
a polynomial expansion

$$
\begin{aligned}
\bar{x}_{i}-x_{i} & =A_{0}+A_{1}^{1,0}\left(x_{i}-x_{0}\right)+A_{1}^{0,1}\left(y_{i}-y_{0}\right)+\ldots \\
& =A_{0}+\sum_{m=1}^{k / 2-1} \sum_{\substack{\alpha, \beta=0, \alpha+\beta=m}}^{m} A_{m}^{\alpha, \beta}\left(x_{i}-x_{0}\right)^{\alpha}\left(y_{i}-y_{0}\right)^{\beta}
\end{aligned}
$$

of measured $\bar{x}_{i}, \bar{y}_{i}$ coordinates at Ep2 over cross-moments of the coordinate differences $x_{i}-x_{0}, y_{i}-y_{0}$ at Ep1 of a standard frame, where the index $i=0$ again refers to the target. The equation for $y$ has a similar structure. A residual of conditional equations at $i=0$ is treated as a proper motion

$\mu_{x}=\bar{x}_{0}(\mathrm{Ep} 2)-x_{0}(\mathrm{Ep} 1)-A_{0}$.

Note that all coefficients $A_{m}^{\alpha, \beta}$ with $m>0$ have vanished since Eqs. (7) are written for a chosen $i=0$ star. This result is completely equivalent to Eq. (6) since, multiplying Eq. (7) by $a_{i}$ and then summing up over $i$, in view of conditions (5) we find that $A_{0}=N^{-1} \sum a_{i}\left(\bar{x}_{i}-x_{i}\right)$. Thus, symmetrization allows both reduction of the image motion and exclusion of all geometric distortions (their change between Ep1 and Ep2) up to and including the polynomial degree of $\alpha+\beta=k / 2-1$. In contrast, if the reduction model (7) includes all modes $m \leq k / 2-1$ with no omissions, the least squares solution of systems (7) and ((4)-(5)) are equivalent. It means that, though the use of model (7) is aimed at correcting geometric distortions, it reduces the image motion equally well. For example, a 6 parameter linear model normally used in proper motion studies (e.g. Pravdo \& Shaklan 1996) in fact performs the $k=4$ order symmetrization.

\section{Differential positions}

A one-hour series of $j=1,2 \ldots 72$ frames obtained near the meridian was used for a detailed analysis. A frame $j=1 \mathrm{ob}-$ tained near the meridian was chosen as the standard "epoch 1 " frame. In this frame, assuming some star as a target $i=0$ and using Eqs. (5), we computed a set of weights $a_{i}$ with which the target star positions $W_{j}$ at each other $j$ frame ("epoch 2 ") were calculated. Computations were performed at $k=2,4 \ldots 12$ and at reference field radii $R$ from $0.2^{\prime}$ to $1.1^{\prime}$, whereupon the whole procedure was repeated, considering in turn each other star as a target. Since the actual image motion for so short a time is zero, the displacements computed, of course, represent errors of $W_{x}, W_{y}$ determination. Because a noticeable part of these errors is caused by centroiding errors of reference stars, we performed iterative refinement of positions $\bar{x}_{i}, \bar{y}_{i}$. For this purpose we calculated the preliminary $W_{x}, W_{y}$ values with $k=6$ and near optimal $R=0.6^{\prime}$. These displacements taken with reference to their average $\left\langle W_{x}\right\rangle,\left\langle W_{y}\right\rangle$ over the whole series of $j=1,2 \ldots 72$ frames were treated as centroiding errors and therefore subtracted from the $\bar{x}_{i}, \bar{y}_{i}$ coordinates of the corresponding $i$ th star. While reducing random errors of referencestar positions, these iterations introduce an extra atmospheric dependent and spatially-correlated bias. For this reason only a small improvement in the final precision was obtained and only at the first iteration; no improvement was found for $k=2$ at all.
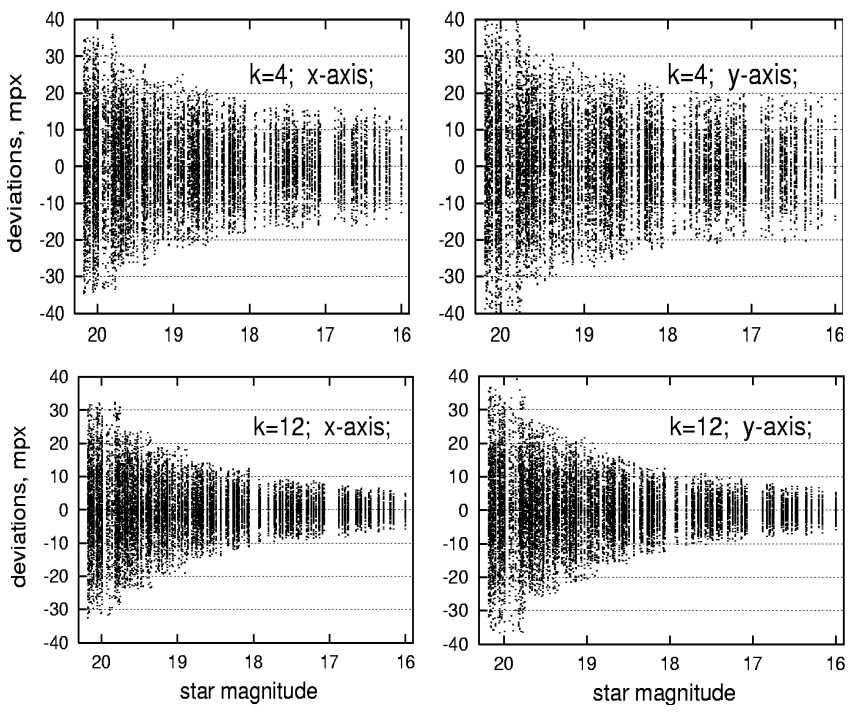

Fig. 1. Astrometric deviations $W_{j}-\langle W\rangle$ of each frame's star position $W_{j}$ from its average position $\langle W\rangle$ as a function of instrumental magnitude; plots correspond to a $0.8^{\prime}$ reference field radius and reduction parameters $k=4$ and $k=12$.

\subsection{Astrometric error components}

Figure 1 represents astrometric errors as residuals $W_{j}-\langle W\rangle$ of each frame's star position $W_{j}$ from its average $\langle W\rangle$ over all 72 frames. The distribution of residuals computed with $R=$ $0.8^{\prime}, k=4$, and $k=12$ is seen to be symmetric and dependent on the star brightness, which is especially contrasted at $k=12$. Errors of $W_{y}$ are slightly larger due to the image elongation along the $y$-axis. Note the difference of point scatter at $k=4$ and $k=12$, which were obtained with use of identical sets of reference stars.

The measured variance $\Delta_{k}^{2}$ of each star position computed with application of some $k$ order was found as $\Delta_{k}^{2}=$ $\overline{\left(W_{j}-\langle W\rangle\right)^{2}}$. Also $\Delta_{k}$ defines a precision of $W$ value zeropoints, which are set so that $W(\mathrm{Ep} 1)=0$. The $W(\mathrm{Ep} 1)$ values are thus shifted randomly from $\langle W\rangle$ by about $\Delta_{k}$ rms.

The estimates of $\Delta_{k}^{2}$ plotted as a function of $R$ show a large scatter of points (Fig. 2a); however, a clear dependence on $k$, $R$, and, of course, on the star brightness (the plot is built for 16.5 mag star) is seen. To model this dependence, we decomposed the $\Delta_{k}^{2}$ value as a sum

$\Delta_{k}^{2}=\sigma_{\mathrm{at}}^{2}+C^{2} \sigma_{\mathrm{rf}}^{2}+\eta^{2} \varepsilon_{0}^{2}$

of atmospheric component (3) that at large number of reference stars is approximated by the expression (Eqs. (36)-(38) of Paper I)

$\sigma_{\text {at }}=B_{k}\left(R / 1^{\prime}\right)^{b_{k}}$

with parameters $B_{k}$ and $b_{k}$ dependent on $k$ and considered here as free model parameters; the centroiding noise of the reference field

$\sigma_{\mathrm{rf}}=N^{-1} \sqrt{\sum a_{i}^{2} \varepsilon_{i}^{2}}$

where $\varepsilon_{i}$ is given by Eq. (2), and the third component $\eta \varepsilon_{0}$ which represents the centroid precision for the target star. Coefficient 

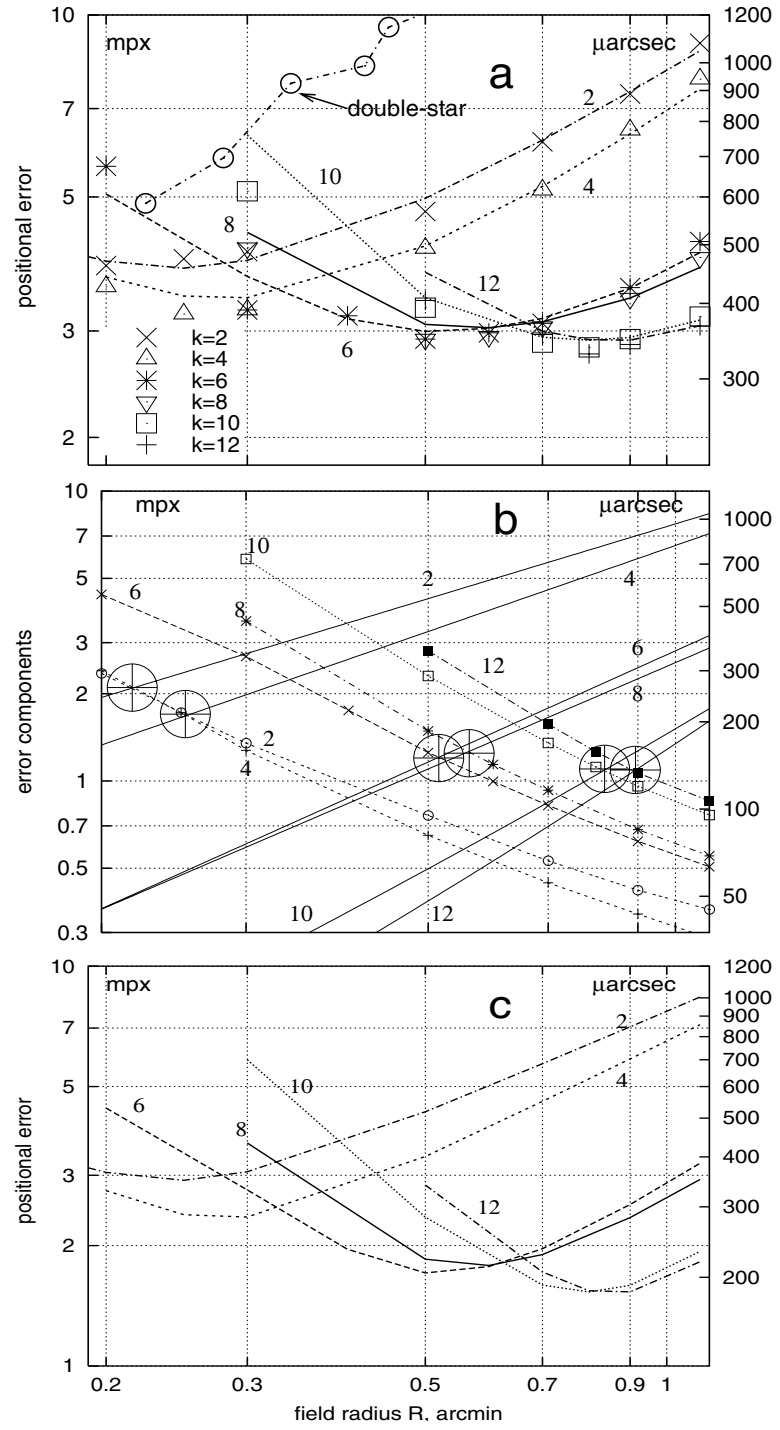

Fig. 2. Dependence of the total astrometric error and its components on $R$ at $k=2,4 \ldots 12$. a) The total error $\Delta_{k}^{2}$ of $16.5 \mathrm{mag}$ star observation: measured point estimates (dots) and approximation (9) (lines); errors in double-star separation measurements are shown with a shift: a real curve is a factor 2 upward. b) Reference field components: $\sigma_{\text {rf }}$ (dashed lines with different symbols) and $\sigma_{\text {at }}$ (solid lines); large cicles mark points of line intersections at optimal radii $R_{\mathrm{opt}}$. c) The cumulative reference field contribution $\Delta_{\mathrm{a}+\mathrm{r}}$. All plots refer to right ascension.

$C<1$ is introduced to reflect the decrease in $\sigma_{\text {rf }}$ component produced by the iterative refinement of star positions, and $\eta \geq 1$ is the ratio of the true centroid error to its photon-limited estimate (2); it was treated as a free model parameter for each 0.5 mag bin of magnitudes. The solution of Eq. (9) produced $B_{k}$ and $b_{k}$ values, $C \approx 0.7, \eta \approx 1$ for stars fainter than $17 \mathrm{mag}$ and, due to PSF profile imperfection, $\eta^{2} \approx 1.2-1.3$ for brighter images.

Figure 2b shows dependence of components $C \sigma_{\text {rf }}$ (units: $0.001 \mathrm{px}=1$ millipixel, hereafter $\mathrm{mpx} ; 1 \mathrm{mpx}=125 \mu$ as (microarcsec)) and $\sigma_{\text {at }}$ on $R$ at each parameter $k$ value. The observed dependencies qualitatively match theoretic behavior of the plots shown in Fig. 15 of Paper I and corresponding to $D=10 \mathrm{~m}$ and $T=10 \mathrm{~min}$. At narrow fields, the dominant
Table 1. Parameters $B_{k}\left[\mu\right.$ as] and $b_{k}$ calculated for $X$ and $Y$ and scaled from Fig. 15 of Paper I.

\begin{tabular}{|c|c|c|c|c|c|c|}
\hline$k$ & $\begin{array}{r}B_{k} \\
\text { Com }\end{array}$ & $\begin{array}{r}b_{k} \\
\ln X\end{array}$ & $\begin{array}{r}B_{k} \\
\text { Com }\end{array}$ & $\begin{array}{r}b_{k} \\
\text { in } Y\end{array}$ & $\begin{array}{r}B_{k} \\
\text { From }\end{array}$ & $\begin{array}{r}b_{k} \\
\text { aper I }\end{array}$ \\
\hline 2 & 1380 & 0.9 & 1260 & 0.8 & 1190 & 0.64 \\
\hline 4 & 780 & 1.0 & 1010 & 1.0 & 806 & 1.69 \\
\hline 6 & 340 & 1.3 & 420 & 1.2 & 186 & 1.63 \\
\hline 8 & 310 & 1.2 & 370 & 1.1 & 161 & 1.83 \\
\hline 10 & 180 & 1.8 & 230 & 1.4 & 84 & 1.66 \\
\hline 12 & 160 & 1.9 & 200 & 1.6 & 76 & 1.84 \\
\hline
\end{tabular}

component is $\sigma_{\text {rf }}$, which decreases with $R$ following an approximate dependence (Paper I)

$\sigma_{\mathrm{rf}}=\frac{F W H M}{2.36 R \sqrt{\pi n}} \frac{k}{4}$,

where $n$ is the total light from a $1 \times 1^{\prime}$ reference field, $R$ is given in minutes of an arc, $k / 2$ is supposedly even, and star number $N \gg N_{\min }$. For small $N \simeq 3-5 N_{\min }$, the actual values (11) of $\sigma_{\text {rf }}$ are systematically a factor 1.5-2 above the estimate (12). At still lower $N$, the curves for $\sigma_{\text {at }}$ are not built. At $R \simeq 1^{\prime}$ the value of $\sigma_{\text {at }}$ considerably exceeds $\sigma_{\text {rf }}$.

A twinning of curves in Fig. 2 related to pairs $k=(2,4)$, $(6,8)$, and $(10,12)$ is a specific feature of densely populated fields since a high sky star density favors reduction due to the field averaging effects. At $k$ that is not a multiple of 4 , the values of $\sigma_{\text {at }}$ and $\sigma_{\text {rf }}$ are therefore close to approximate estimates (10) and (12) computed with $k+2$. Just for illustration of the field averaging effect, Fig. 2a reproduces the dependency of astrometric error for measurements of $\bar{x}_{0}-\bar{x}_{i}$ separating two stars of approximately equal brightness. The dependence observed implies that to obtain good astrometric precision, a separation $R$ between two stars should be less than $0.1^{\prime}$; at larger $R$ the accuracy degrades very rapidly. This is an example of a marginal single-star reference field, but note that $W_{x}$ positions (4) are composed of $\bar{x}_{0}-\bar{x}_{i}$ unit differences averaged over the field.

Table 1 compares the current parameter $B_{k}$ and $b_{k}$ estimates for VLT and their expectations for Chilean observatories (Cerro Tololo, Paranal, and San Pedro Martir) at typical atmospheric conditions. This data was found by scaling plots from Fig. 15 of Paper I, given for a $D=10 \mathrm{~m}$ and $T=10 \mathrm{~min}$, to the current values using a relation $B_{k}^{2} \sim$ (airmass) $\times T^{-1} D^{-3}$ (Eq. (37) of Paper I). Results given in the last column of Table 1 generally agree with observed values except some differences commented on below. By definition (10), coefficient $B_{k}$ is equal to $\sigma_{\text {at }}$ at $R=1^{\prime}$. Its measured value is approximately equal to the scaled expectation at $k=2$ and $k=4$ but, starting from $k=6$, is a factor 2-3 higher. This may indicate an actual intensity of atmospheric turbulence at high altitudes that, according to the discussion in Paper I, may easily show such fluctuations. A smaller than expected measured value of coefficient $b_{k}$ at $k=4$, 6 , and 8 implies a higher value of $\sigma_{\text {at }}$ value at small $R$. It is unlikely that it is of atmospheric origin, but it is probably due to some effects caused by the active optics performance.

Let us consider a sum $\Delta_{\mathrm{a}+\mathrm{r}}^{2}=\sigma_{\mathrm{at}}^{2}+C^{2} \sigma_{\mathrm{rf}}^{2}$ that determines the properties of a noise from the reference field alone. This component (Fig. 2c) has a typical parabola-like shape with a 
Table 2. Astrometric error components (mpx) and optimal field radii $R_{\text {opt }}$ at $k=2,4 \ldots$ The last column contains data averaged over $k$; centroid error $\eta \varepsilon_{0}$ is given for the brightest stars.

\begin{tabular}{|c|c|c|c|c|c|c|c|}
\hline & \multicolumn{6}{|c|}{$k$} & \multirow[b]{2}{*}{$\langle k\rangle$} \\
\hline & 2 & 4 & 6 & 8 & 10 & 12 & \\
\hline & \multicolumn{6}{|c|}{$x$, right ascension } & \\
\hline$\sigma_{\text {at }}$ & 2.1 & 1.7 & 1.3 & 1.3 & 1.1 & 1.1 & - \\
\hline$C \sigma_{\mathrm{rf}}$ & 2.1 & 1.7 & 1.3 & 1.3 & 1.1 & 1.1 & - \\
\hline$\eta \varepsilon_{0}$ & 1.9 & 1.9 & 1.9 & 1.9 & 1.9 & 1.9 & 1.9 \\
\hline$\Delta_{\mathrm{a}+\mathrm{r}}$ & 3.0 & 2.4 & 1.8 & 1.9 & 1.6 & 1.6 & $\Delta_{\langle\mathrm{a}+\mathrm{r}\rangle}=1.1$ \\
\hline \multirow[t]{2}{*}{$\Delta_{k}$} & 3.5 & 3.0 & 2.5 & 2.5 & 2.4 & 2.4 & $\Delta_{\langle k\rangle}=2.2$ \\
\hline & \multicolumn{6}{|c|}{$y$, declination } & \\
\hline$\sigma_{\text {at }}$ & 2.8 & 2.0 & 1.5 & 1.6 & 1.4 & 1.4 & - \\
\hline$C \sigma_{\mathrm{rf}}$ & 2.8 & 2.0 & 1.5 & 1.6 & 1.4 & 1.4 & - \\
\hline$\eta \varepsilon_{0}$ & 2.2 & 2.2 & 2.2 & 2.2 & 2.2 & 2.2 & 2.2 \\
\hline$\Delta_{\mathrm{a}+\mathrm{r}}$ & 4.0 & 2.8 & 2.1 & 2.2 & 2.0 & 1.9 & $\Delta_{\langle\mathrm{a}+\mathrm{r}\rangle}=1.4$ \\
\hline$\Delta_{k}$ & 4.6 & 3.6 & 3.0 & 3.1 & 2.9 & 2.9 & $\Delta_{\langle k\rangle}=2.6$ \\
\hline$R_{\mathrm{opt}}$ & $0.22^{\prime}$ & $0.28^{\prime}$ & $0.5^{\prime}$ & $0.55^{\prime}$ & $0.8^{\prime}$ & $0.9^{\prime}$ & - \\
\hline
\end{tabular}

minimum at the point $R_{\mathrm{opt}}$ where $\sigma_{\mathrm{at}}^{2}=C^{2} \sigma_{\mathrm{rf}}^{2}$. Note a gradual decrease in the $\Delta_{\mathrm{a}+\mathrm{r}}$ value when applying large $k$ and a corresponding increase in $R_{\mathrm{opt}}$. In our case, the minimum is achieved at $k=10,12$ and $R \sim 0.9^{\prime}$, where $\Delta_{\mathrm{a}+\mathrm{r}}=1.59 \mathrm{mpx}$ for right ascension and $\Delta_{\mathrm{a}+\mathrm{r}}=1.91 \mathrm{mpx}$ for declination. The total astrometric error $\Delta_{k}$ changes with $R$ similarly and also has a minimum at $R=R_{\text {opt }}$.

Centroid errors $\eta \varepsilon$ for the brightest unsaturated stars of $16 \mathrm{mag}$ are equal to $1.86 \mathrm{mpx}$ ( $230 \mu \mathrm{arcsec})$ for the right ascension and $2.22 \mathrm{mpx}(280 \mu \mathrm{arcsec})$ for declination and are on the order of best attainable $\Delta_{\mathrm{a}+\mathrm{r}}$. The atmospheric error components, $\Delta_{k}$ value for these stars and $R_{\text {opt }}$ for each $k$, are listed in Table 2.

Note that for any arbitrary $k$ value (or equivalent order $m=k / 2-1$ of a polynomial model (7)), one can always find a field size $R=R_{\text {opt }}$ at which $\sigma_{\text {at }}=C \sigma_{\mathrm{rf}}$ and where atmospheric image motion represents less than half of the total variance (9). Astrometric measurements thus are never atmospherically limited provided that the reduction is performed with $R_{\text {opt }}$. This conclusion is also valid for the sky areas with a poor sky star density as discussed in Sect. 5 .

\subsection{Averaging over modes}

Positions $W_{k}$ calculated with different $k$ and different reference field radii $R_{\mathrm{opt}}$ are related to distinctive sets of stars and hence have almost uncorrelated components $\sigma_{\mathrm{rf}}$. Atmospheric errors $\sigma_{\text {at }}$ computed with different $k$ (modes $m$ in Eq. (3)) are also weakly correlated since the effective turbulent layer heights $h$ depend on $k$. Thus, while at $k=2$ all turbulent layers from 5 to $20 \mathrm{~km}$ heights contribute equally to the variance of $W_{k}$, the use of $k=12$ effectively cuts off turbulence contribution from $h<20 \mathrm{~km}$ (Fig. 12 of Paper I). It is natural therefore to average positions $W_{k}$ with weights $P_{k}=1 / \Delta_{\mathrm{a}+\mathrm{r}}^{2}$ calculated from Table 1:

$W_{\langle k\rangle}=\sum_{k} P_{k} W_{k} / \sum_{k} P_{k}$.

The total weight of the average is $\sum P_{k}=4.0 P_{12}$. However, taking almost equal size of reference fields for pairs $k=(2,4)$,

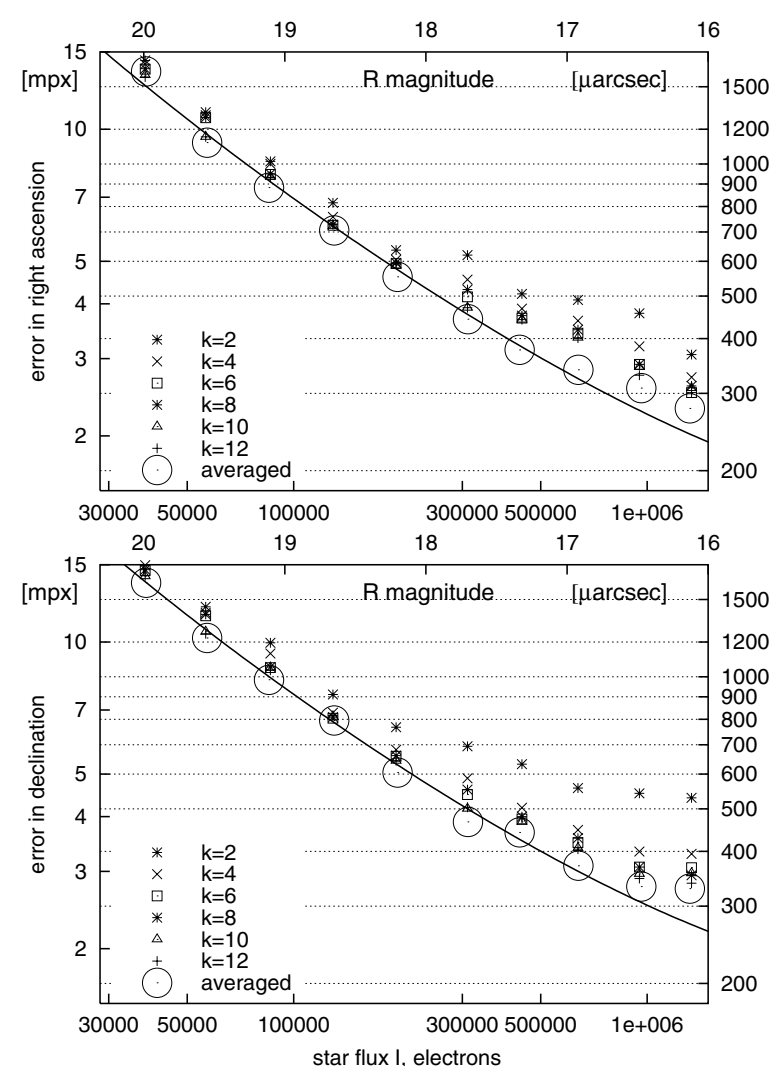

Fig. 3. Astrometric error as a function of the target flux $I$. Different dots $-\Delta_{k}$ at $k=2,4 \ldots 12$; open circles - error $\Delta_{\langle k\rangle}$ of positions averaged over modes; solid line - approximation (14) of $\Delta_{\langle k\rangle}$ assuming $\eta=1$ and with $\varepsilon_{0}$ given by Eq. (2). Upper panel - right ascension, lower-declination.

$(6,8)$, and $(10,12)$ into account, the effective weight is instead $P_{\text {eff }} \simeq 2.0 P_{12}$. The variance of weighed positions (13) is

$\Delta_{\langle k\rangle}^{2}=\Delta_{\langle\mathrm{a}+\mathrm{r}\rangle}^{2}+\eta^{2} \varepsilon_{0}^{2}$

with a new reference field component

$\Delta_{\langle\mathrm{a}+\mathrm{r}\rangle}^{2}=\left.\frac{P_{12}}{P_{\text {eff }}} \Delta_{\mathrm{a}+\mathrm{r}}^{2}\right|_{\text {at } k=12}$

decreased almost twice (Table 2) in comparison to its value $\Delta_{\mathrm{a}+\mathrm{r}}^{2}$ at $k=12$.

Figure 3 shows errors $\Delta_{k}$ (at $R=R_{\text {opt }}$ ) and $\Delta_{\langle k\rangle}$ as a function of the target brightness. These estimates were obtained from the convergence of the measured positions $W_{k}$ and $W_{\langle k\rangle}$ at different frames and after applying a small correction for color effects (Sect. 6). The model dependence (14) plotted at $\eta=1$ (solid curve) predicts the measured estimates till $R<17 \mathrm{mag}$ well. At the brighter end the actual errors exceed their values expected at $\eta=1$ due to the non-Poisson noise in star profiles $\left(\eta^{2} \sim 1.2\right)$. The astrometric error $\Delta_{\langle k\rangle}$ is roughly equal to $\varepsilon_{0}$ at $R>17 \mathrm{mag}$ and to $1.5 \varepsilon_{0}$ for brighter stars. An error component introduced by incorrect tying to the reference frame is thus rather small.

\section{Astrometric error at low sky star density}

The results obtained above for high sky star density frames can be used to predict astrometric performance of VLT at other 


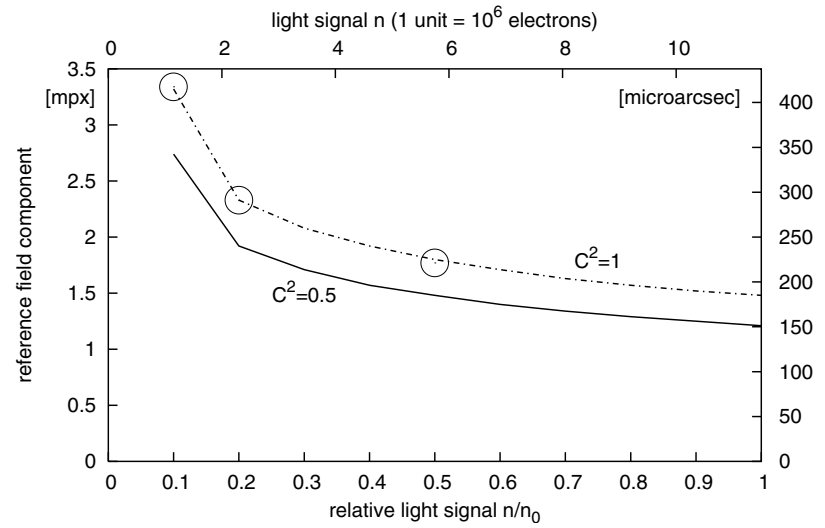

Fig. 4. An error of referencing $\Delta_{\langle\mathrm{a}+\mathrm{r}\rangle}$ as a function of reference field total light $n$ : based on the scaling expression (16) at $C^{2}=0.5$ (solid curve), the same at $C^{2}=1$ (dashed line), and found from a direct reduction of a sparsed original sample of stars (open circles).

sky regions. The most important parameter necessary for this purpose is the integral light flux $n$ registered from reference stars. Its average value for our OGLE field is equivalent to $n_{0}=12 \times 10^{6} \mathrm{e}^{-}$from a square minute of arc $(60$ stars brighter than $R=20$ mag at $1 \times 1^{\prime}$ area). It essentially affects the $\sigma_{\text {rf }}$ value, which according to Eq. (12) varies as $\sigma_{\text {rf }} \sim n^{-1 / 2}$, while $\sigma_{\text {at }}$ does not depend on $n$ (10). At $n \neq n_{0}$ the curves in Fig. 1 relevant to the $\sigma_{\mathrm{rf}}$ component are shifted, moving the point $R_{\mathrm{opt}}$ to a new position $R_{\mathrm{opt}}^{\prime}=\theta R_{\mathrm{opt}}$.

Using Eqs. (10) and (12) for scaling $\sigma_{\text {at }}$ and $\sigma_{\text {rf }}$ components at a new value of $R_{\mathrm{opt}}^{\prime}$ from a requirement of a balance $\sigma_{\text {at }}=C \sigma_{\mathrm{rf}}$, we find $\theta=\left[\left(\omega C^{2} n_{0}\right) /\left(C_{0}^{2} n\right)\right]^{1 /\left(2 b_{k}+2\right)}$ where $C_{0}^{2}=0.5$. Note that Eq. (12) systematically gives underestimated $\sigma_{\text {rf }}$ value for frames with a small number of reference stars $N \sim(3-5) N_{\min }$. In view of this we introduce the parameter $\omega \geq 1$ equal to the ratio of the actual $\sigma_{\text {rf }}$ value given by Eq. (11) to its approximate estimate (12). For the sample analyzed, $\omega \simeq 1$ at $n \geq 0.2 n_{0}$, while at low $n=0.1 n_{0}$ it grows from 1 to 2.5 when $k$ changes from 4 to 12 . For $R_{\mathrm{opt}}$ and $b_{k}$ relevant to some $k$ we obtain $R_{\text {opt }}^{\prime}=\left[\left(\omega C^{2} n_{0}\right) /\left(C_{0}^{2} n\right)\right]^{1 /\left(2 b_{k}+2\right)} R_{\text {opt }}$, hence the expected error of referencing

$\Delta_{\mathrm{a}+\mathrm{r}}^{\prime}=\left[\left(\omega C^{2} n_{0}\right) /\left(C_{0}^{2} n\right)\right]^{b_{k} /\left(2 b_{k}+2\right)} \Delta_{\mathrm{a}+\mathrm{r}}$

Based on this expression and using parameter values listed in Tables 1 and 2, we calculated the expected values of $\Delta_{\mathrm{a}+\mathrm{r}}^{\prime}$ at $n=0.1 \ldots 1.0 n_{0}$ in two variants with $C^{2}=0.5$ and with $C^{2}=1$. The resulting error $\Delta_{\langle k\rangle}$ that corresponds to the error (15) of the average over modes is shown in Fig. 4.

To control our scaling approach, we performed direct computations of $\Delta_{\langle k\rangle}$ using the original but artificially sparsed star sample and with no iterative improvement of reference star positions $\left(C^{2}=1\right)$. Results (open circles) match the estimates (16) well. Note that at $n \geq 0.2 n_{0}$ a good referencing at the 1.5-2 mpx level is maintained, which is comparable to the bright star centroid precision. At $n \sim 0.1 n_{0}$ due to the decrease in a light signal from reference stars, the accuracy of the referencing degrades sharply. Besides, at highest $k=12$ order, the optimum field size $2 R_{\mathrm{opt}}^{\prime}=3.2-3.5^{\prime}$ is near the CCD size.
Table 3. Centroid precision and astrometric error ( $\mu$ as) at a $10 \mathrm{~min}$ total exposure and different $n / n_{0}$ for reduction versions with $C^{2}=1.0$ and 0.5. A ratio of $\sigma_{\mathrm{at}}^{2}$ to the total error (last column) and reference field contribution $\Delta_{\mathrm{a}+\mathrm{r}}^{\prime}$ (last line) are also given.

\begin{tabular}{r|r|rrr|rrr|r}
\hline \hline & Centr & \multicolumn{3}{|c|}{ Red. with $C^{2}=1$} & \multicolumn{2}{|c|}{ Red. with $C^{2}=0.5$} & $\sigma_{\text {at }}^{2}$ \\
ratio & error & \multicolumn{3}{|c|}{$n / n_{0}$} & \multicolumn{3}{|c}{} & \\
mag & $\eta \varepsilon$ & 0.1 & 0.2 & 1.0 & 0.1 & 0.2 & 1.0 & $\%$ \\
\hline 20 & 300 & 308 & 304 & 301 & 305 & 302 & 301 & 0 \\
19 & 167 & 181 & 174 & 170 & 177 & 172 & 169 & 1 \\
18 & 98 & 120 & 110 & 103 & 114 & 106 & 101 & 3 \\
17 & 65 & 95 & 81 & 72 & 87 & 77 & 70 & 6 \\
16 & 42 & 81 & 64 & 52 & 71 & 58 & 49 & 13 \\
15 & 26 & 75 & 56 & 41 & 63 & 48 & 36 & 24 \\
14 & 16 & 72 & 52 & 35 & 60 & 44 & 30 & 35 \\
\hline$(0)$ & $(0)$ & 70 & 49 & 31 & 58 & 40 & 25 & 50 \\
\hline
\end{tabular}

At very low $n$ the star number in the area of the optimal radius $R=R_{\text {opt }}^{\prime}$ may be smaller than $N_{\text {min }}$ and so insufficient for implementation of the desired $k$ order. This is extremely unfavorable since it forces one to use lower $k$ or, even worse, perform reduction without optimal $R$. Using the above expressions for $R_{\mathrm{opt}}^{\prime}$ and $N_{\mathrm{min}}$ and assuming $C=C_{0}$, we find that a low limit of reference star density necessary to implement some $k$ symmetry order is $n_{\min }=n_{0}\left[k(k+2) /\left(8 \pi n_{0} R_{\mathrm{opt}}^{2}\right)\right]^{1+1 / b_{k}} \omega^{-1 / b_{k}}$ Assuming $\omega \approx 2-3$ and taking $b_{k}$ and $R_{\text {opt }}$ values from Tables 1 and 2 , we find that approximately $n_{\min } \simeq 0.01 n_{0}$ at any $k$ from 4 to 12 . This integral star density (about 0.6 stars brighter than $R=20 \mathrm{mag}$ per $1 \times 1^{\prime}$ area) is typical of the Galactic pole (Allen 1973).

The inequality $N>N_{\min }$ can be easily restored in any case by incorporation of some extra faint stars as reference. This does not degrade the precision; on the contrary, the total flux $n$ will only increase and, according to Eq. (12), $\sigma_{\mathrm{rf}}$ improves. We conclude therefore that even at low sky star density the key problem of astrometric error improvement is related to the telescope light signal limitation but not to the atmospheric image motion (see Table 3, last column).

Table 3 gives the centroid precision and astrometric error (14) for 14-20 mag (R) stars expected from a reduction of a series of frames with a total $T=10 \mathrm{~min}$ exposure and of 30-50 min (depending on observation mode) duration. For bright 14-16 mag stars we adopted $\eta^{2}=1.3$, for $17 \mathrm{mag}$ we used $\eta^{2}=1.2$, and for fainter stars $\eta=1$. The estimates are given for reduction versions corresponding to $C^{2}=1$ and $C^{2}=0.5$. In the last line, the total reference field contribution $\Delta_{\mathrm{a}+\mathrm{r}}^{\prime}$ is given, which is equal to the limiting astrometric precision of very bright stars $(\varepsilon=0)$. At high sky star density, this limit is $25 \mu$ as per 10 min exposure. The last column contains a ratio of atmospheric image motion to the total error; as discussed above, this portion cannot exceed $50 \%$ by the definition of the optimal field size.

For the brightest stars, thus, the expected accuracy of observations in the Galactic bulge is equal to $30-50 \mu$ as at 10 min exposures, degrading to $50-80 \mu$ as at $n \sim 0.1-0.2 n_{0}$ (2-4 $\times 10^{4}$ stars to $R=20 \mathrm{mag}$ per square degree, which corresponds to galactic latitudes of $20-30^{\circ}$; Allen 1973). For stars fainter than $R>17 \mathrm{mag}$, the error does not depend on the 
characteristics of the reference field due to a small light signal from the target.

\section{DCR and residual chromatism of LADC}

The effects of DCR was considered elsewhere. In particular, Monet et al. (1992) studied restriction of the accuracy of proper motions derived from the reduction of frames obtained at different hour angles. In this study we use a mathematical approach similar to the one used by Monet et al. (1992). However, we have to consider the joint influence of an atmosphere and of the chromatic corrector LADC (Avila et al. 1997) used at VLT to improve the image quality.

It is known that the relative displacement $S$ of two monochromatic images with wavelengths $\lambda_{1}$ and $\lambda_{2}$ is proportional to a tangent of zenith distance $z: S=\delta \tan z$ where $\delta$ is the coefficient of atmospheric dispersion dependent on a difference $\lambda_{1}-\lambda_{2}$. The corrector reduces this displacement, incorporating a negative equivalent displacement $-(\delta-d) \tan z$ where $d$ is the residual error of the corrector. The observed relative displacement of the images in a vertical direction therefore is $d \tan z$. When a series of frames is obtained at VLT, the corrector is preset at some fixed zenith distance $z_{\mathrm{L}}$ and this setting does not change. Therefore

$S=\delta\left(\tan z-\tan z_{\mathrm{L}}\right)+d \tan z_{\mathrm{L}}$

If $\lambda_{1}$ is an effective wavelength of a target star and $\lambda_{2}$ is effective wavelength of reference field, the measured displacements $S_{x}, S_{y}$ are equal to the $S$ projection at the CCD coordinate system axis ( $x$ : opposite to right ascension, $y$ : along the declination)

$$
S_{x}=S \sin \gamma+S_{x 0}, S_{y}=S_{0}-S \cos \gamma+S_{y 0},
$$

where $\gamma$ is the angle formed by a direction to zenith and the $y$ axis, $S_{0}$ is the $S$ value in a meridian, and $S_{x 0}, S_{y 0}$ are zeropoints.

The values of $\delta$ and $d$ were found from a least square solution of Eq. (18) for each star. As input, we used the positions of stars in all 280 frames that were calculated with $k=10$ and $R=0.8^{\prime}$. The results are shown in Fig. 5 as a function of relative color $v-r-\overline{(v-r)}$ where $\overline{v-r}$ is the mean instrumental color index of a local star group used as the reference for some target star. The distribution of point estimates in plots is approximated well by linear functions

$\delta=-2.6+(233 \pm 8)[v-r-\overline{(v-r)}]$

$d=-(11.0 \pm 0.9)[v-r-\overline{(v-r)}]$,

where values are expressed in mpx and correspond to atmospheric pressure $744 \mathrm{mb}$, temperature $11^{\circ}$, and $11 \%$ humidity. The residual scatter of points in Fig. 5 is $\sigma(\delta)=11.9 \mathrm{mpx}$ for $\delta$ and $\sigma(d)=1.3 \mathrm{mpx}$ for $d$, and a factor 4-5 exceeds formal errors of $\delta$ and $d$ value determination. It can only be partially explained by the errors of color determination $( \pm 0.02 \mathrm{mag})$. Most likely, this scatter is intrinsic and not can be adequately modelled using only $v-r$ colors.
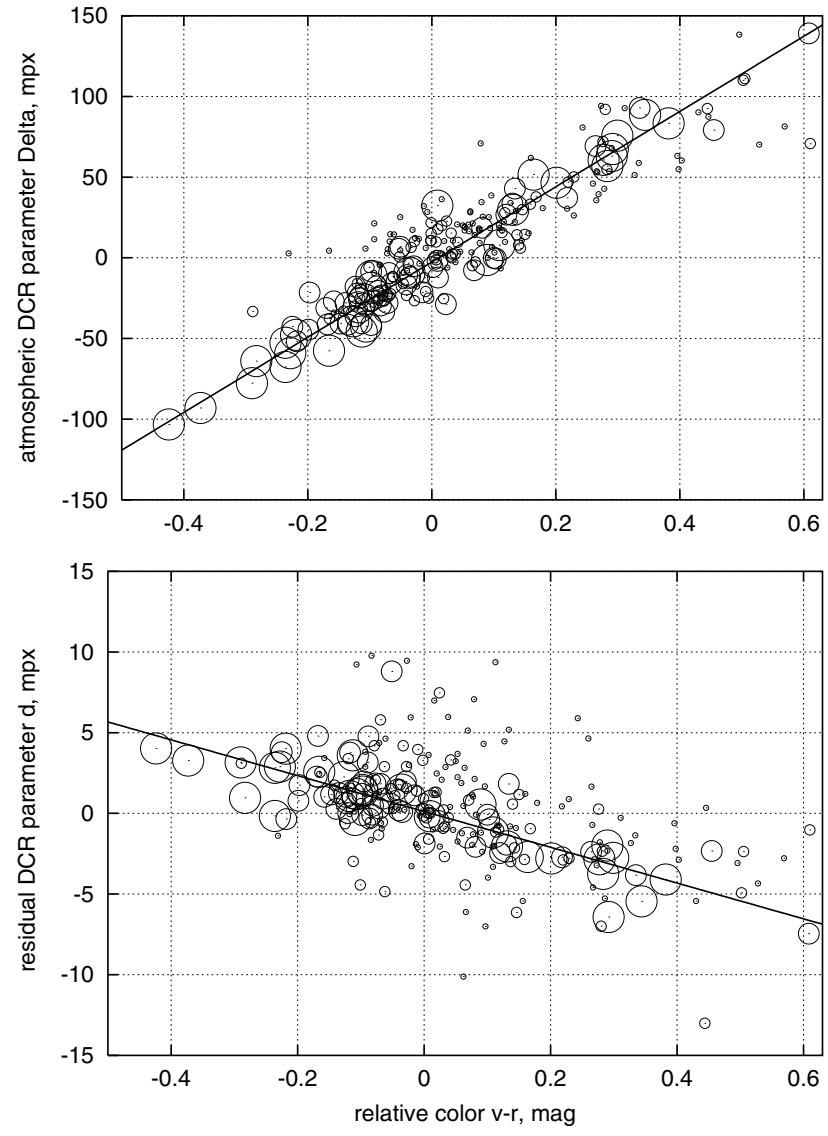

Fig. 5. Atmospheric dispersion coefficient $\delta$ (upper panel) and the residual LADC bias $d$ (lower) as a function of relative star color $v-r-\overline{(v-r)}$. The size of circles is proportional to the star brightness (from $3 \times 10^{4}$ to $1.2 \times 10^{6} \mathrm{e}^{-}$); solid lines - approximation (19).

To check the reliability of the results, we performed a control computation of a chromatic refractive index $\rho$ using the expression

$\rho=346 \operatorname{mas}^{\lambda_{\max }} \int_{\lambda_{\min }}^{\lambda_{\max }} \lambda^{-2} T(\lambda) I(\lambda) \mathrm{d} \lambda / \int_{\lambda_{\min }}^{\lambda_{\max }} T(\lambda) I(\lambda) \mathrm{d} \lambda$

given by Pravdo \& Shaklan (1996). The integration is performed within the wavelengths limits $\lambda_{\min }, \lambda_{\max }$ defined by a combined transmission $T(\lambda)$ of the filter and CCD, and $I(\lambda)$ is the star flux function of wavelength. This expression is normalized to $0^{\circ}$ and a pressure of $1013 \mathrm{mb}$. We calculated $\rho$ for stars with blackbody temperatures corresponding to G0 and M0 spectral types, where $V-R$ colors 0.52 and 1.1 are typical of the analyzed sample of stars. The difference in $\rho$ values computed for these spectra and reduced to a unit interval of colors is $38 \mathrm{mas} / \mathrm{mag}$ and corresponds to the color coefficient in expression (19) for $\delta$. After reduction to the pressure and temperature at VLT, we obtained $216 \mathrm{mpx} / \mathrm{mag}$, which agrees with the model (19) value within the error limits.

Using LADC reduces the bias caused by atmospheric color effects considerably. At precise presetting of LADC $z_{\mathrm{L}}=z$, the bias $S$ is minimized to $S=d \tan z_{\mathrm{L}}$ with coefficient $d 20$ times smaller in comparison to atmospheric coefficient $\delta$.

Image displacement induced by the DCR effect can be considerable. For example, for a red star with $v-r=1.43$ and 


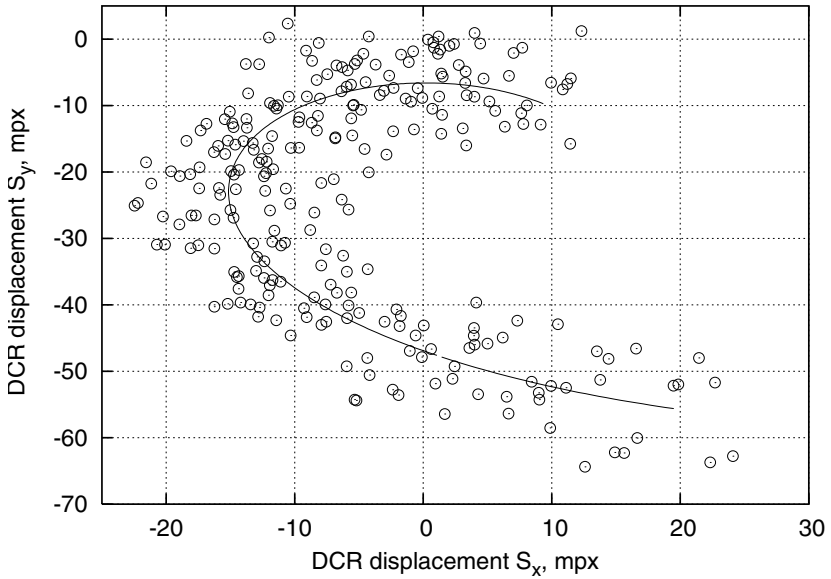

Fig. 6. Chromatic image motion (counter-clockwise and downwards) of a red $R=17.5$ mag star with $v-r=1.43$ over a CCD for 4 h: points - measured positions; solid curve - approximation (18).

local reference field average color $\overline{v-r}=0.82$, the amplitude of the displacement at hour angles $t \sim 3.5$ reaches $60 \mathrm{mpx}$ with an average $20 \mathrm{mpx} /$ hour rate of image motion (Fig. 6). For our observation session, LADC was set at $z_{\mathrm{L}}=45^{\circ}$ while in a meridian $z_{\mathrm{m}}=37.3^{\circ}$.

The DCR effect is a cause of proper motion systematic errors proportional to the difference in $S_{x}, S_{y}$ values related to the median moments of frames representing Ep1 and Ep2. In view of this, observations should be carried out at a minimum difference in hour angles $\Delta t$. Let us estimate the restriction on $\Delta t$ if star colors and coefficients of Eqs. (19) are known. For observations in meridian at $z_{\mathrm{L}}=z_{\mathrm{m}}$, Eq. (17) is reduced to $S=d \tan z_{\mathrm{m}}$. Considering $\gamma \sim t$, from Eqs. (18) one can see that the largest error $\sigma\left(S_{x}\right) \sim \Delta t \sigma(d) \tan z_{\mathrm{m}}$ dependent on $\Delta t$ is expected in $S_{x}$. At the desired accuracy, say $\sigma\left(S_{x}\right)=0.1 \mathrm{mpx}$ and $\sigma(d)$ quoted above, a typical restriction on $\Delta t$ is roughly $20 \mathrm{~min}$, irrespective of a star color. The precision of model (19), thus, is marginal for calibrating near meridian observations obtained with the filter $R_{\text {special }}$.

Restrictions are much stronger for observations at large hour angles. In this case the main error is caused by the $\delta$ term in Eq. (17), and even with use of colors, a good calibration of DCR displacements is possible only at a very strong restriction $\Delta t \leq 1-5 \mathrm{~min}$.

\section{Conclusion}

This study is a first practical test of an astrometric method based on the symmetrization of reference fields. We have shown that, in contrast to the common belief, differential astrometric observations with large telescopes (providing performing an adequate reduction) are not atmospherically limited. At optimal reduction, the photon centroid noise from the reference field is exactly equal to the atmospheric noise. Considering extra cetroiding errors from the target star, the photon noise component always dominates the image motion (Table 3).
This study allows us to predicate that astrometric precision of observations at the VLT is $30-50 \mu$ as for stars of 14-16 mag at $10 \mathrm{~min}$ exposure (a session of observations taking about $40 \mathrm{~min}$ ). This precision is good enough for determining precise parallaxes and proper motions of stars and for examining microlensing effects and exoplanets. Of course, the measured parallaxes and proper motions would be relative. Moreover, since each target star position is measured with reference to its peculiar set of background stars, the zero-points of parallaxes and proper motions are different for different stars. This circumstance, however, is not critical for the purposes of exoplanet and (to a minor extent) for microlensing studies that only require relative astrometric data. If necessary, zero-points can be easily reduced to a common system by iterative procedure.

The most essential restriction for VLT is the saturation of bright images. Also, the sufficiently large light signal necessary for a good referencing can be obtained if observations are restricted to galactic latitudes $20-30^{\circ}$. Atmospheric color effects, generally rather large, can be compensated by relevant calibrations based on star colors and by applying restrictions on hour angles. Long-term systematic errors were not considered in this study and may be a source of additional bias.

Existing filled aperture 8-10 m telescopes are powerful astrometric tools due to effective averaging of atmospheric fluctuations of a phase over the aperture and to providing strong light signals. For larger telescopes, in view of dependences $\varepsilon \sim D^{-1}$, $\sigma_{\text {rf }} \sim D^{-1}$, and $\sigma_{\text {at }} \sim D^{-3 / 2}$, a further increase in the accuracy is expected. Interesting possibilities are allowed by apodization of an entrance pupil (Paper I) and, in particular, by use of adaptive optics that both improves centroiding precision and removes the low-frequency components of the image motion spectrum.

Acknowledgements. I would like to thank Prof. M. Mayor for suggesting this interesting study, Dr. C.Moutou for kindly providing me with CCD frames, and Dr. L. R. Bedin for many helpful remarks on different aspects of this Paper.

\section{References}

Allen, C. W. 1973, Astrophysical quantities (The Atlone Press), University of London

Avila, G., Rupprecht, G., \& Beckers, J. M. 1997, Proc. SPIE, 2871, 1135

Benedict, G. F., McArthur, B. E., Forveille, T., et al. 2002, ApJ, 581, L115

Boden, A. F., Shao, M., \& Van Buren, D. 1998, ApJ, 502, 538

Frink, S., Hekker, S., Lanuhardt, J., et al. 2004, Proc. SPIE, 5491, 1166

Gatewood, D. G. 1987, AJ, 94, 213

Han, I. 1989, A\&A, 97, 607

Lazorenko, P. F., \& Lazorenko, G. A. 2004, A\&A, 427, 1127

Lindegren, L. 1980, A\&A, 89, 41

Louarn, M., Hubin, N., Sarazin, M., \& Tokovinin, A. 2000, MNRAS, 317,535

Monet, D. G., Dahn, C. C., Vrba, F. J., et al. 1992, AJ, 103, 638

Moutou, C., Pont, F., Bouchy, F., \& Mayor, M. 2004, A\&A, 424, L31

Pravdo, S., \& Shaklan, S. 1996, AJ, 465, 264

Safizadeh, N., Dalal, N., \& Griest, K. 1999, ApJ, 522, 512 